Review Article

\title{
Effect of Inulin-Type Carbohydrates on Insulin Resistance in Patients with Type 2 Diabetes and Obesity: A Systematic Review and Meta-Analysis
}

\author{
Mingyue Rao $\left(\mathbb{D},{ }^{1,2}\right.$ Chenlin Gao $\left(\mathbb{D},{ }^{1,3}\right.$ Ling Xu $\left(\mathbb{D},{ }^{3}\right.$ Lan Jiang $\left(\mathbb{D},,^{3}\right.$ Jianhua Zhu $\left(\mathbb{D},{ }^{3}\right.$ \\ Guo Chen $\mathbb{D}^{\mathbb{D}},{ }^{3}$ Betty Yuen Kwan Law $\mathbb{D}^{1},{ }^{1}$ and Yong $X u \mathbb{D}^{1,2}$ \\ ${ }^{1}$ State Key Laboratory of Quality Research in Chinese Medicine, Faculty of Chinese Medicine, Macau University of Science \\ and Technology, Avenida Wai Long, Taipa, Macau, China \\ ${ }^{2}$ Department of Oncology, Affiliated Hospital of Southwest Medical University, Luzhou, Sichuan 646000, China \\ ${ }^{3}$ Department of Endocrinology, Affiliated Hospital of Southwest Medical University, Luzhou, Sichuan 646000, China
}

Correspondence should be addressed to Betty Yuen Kwan Law; yklaw@must.edu.mo and Yong Xu; xywyll@aliyun.com

Mingyue Rao and Chenlin Gao contributed equally to this work and are co-first authors.

Received 17 February 2019; Accepted 28 April 2019; Published 27 August 2019

Academic Editor: Antonio Brunetti

Copyright ( 2019 Mingyue Rao et al. This is an open access article distributed under the Creative Commons Attribution License, which permits unrestricted use, distribution, and reproduction in any medium, provided the original work is properly cited.

\begin{abstract}
Background. Insulin resistance (IR) is a physiological condition related to type 2 diabetes mellitus (T2DM) and obesity, which is associated with high blood insulin and glucose. Inulin-type carbohydrate (ITC) is a kind of fermentable fructan that can reduce glucose and ameliorate IR in an animal model, but the effect in clinical trials is controversial. Objective. The authors conducted a systematic literature review to evaluate the effect of ITC supplementation in ameliorating IR in T2DM and obese patients. Methods. Multiple databases were queried for studies before December 25, 2018, which involved supplementation with ITC in ameliorating IR in T2DM and obese patients. Studies that involved meta-analysis of the body mass index (BMI), fasting plasma glucose (FPG), fasting insulin (FI), HbAlc, homeostatic model assessment IR (HOMA-IR), and quantitative insulin sensitivity check index (QUICKI) of T2DM subjects were included. HOMA-IR and QUICKI were identified as the primary outcomes. A systematic review was performed to evaluate the effect of ITC on IR in obese patients. Results. The database search yielded 25 studies, which met the inclusion criteria; 11 articles were meta-analyzed, and 5 other articles on T2DM and 9 articles on simple obesity were systematically reviewed. Our results did not find ITC supplementation decrease postintervention and reduction data of BMI $(P=0.08)$. However, it can significantly decrease postintervention and reduction data of FPG, FI, HbA1c, and HOMA-IR. Heterogeneity was eliminated by subgroup analysis according to baseline BMI. There was no significant difference in the amelioration of QUICKI between the ITC and control groups. However, the difference was statistically significant and the heterogeneity was eliminated after subgroup analysis according to intakes of ITC. 14 articles for a systematic review found that the results of blood glucose, insulin, and HbAlc were controversial. Only one of the seven studies on simple obesity concluded that ITC intervention significantly ameliorated HOMA-IR, while the other six did not. Conclusion. Supplementation of ITC can ameliorate IR in T2DM, especially in obese T2DM patients, but the effects are controversial in obese patients.
\end{abstract}

\section{Introduction}

Type 2 diabetes mellitus (T2DM) is considered a multifactorial disease, promoted by both genetic and environmental factors, which is characterized by chronic hyperglycemia and insulin resistance (IR) $[1,2]$. The global prevalence of diabetes is estimated by the International Diabetes Federation and indicated that there are 451 million diabetic patients worldwide in 2017, of which T2DM accounts for about 90\% [3]. Diet with low fiber, high fat, and sugar has been linked to obesity [4], 


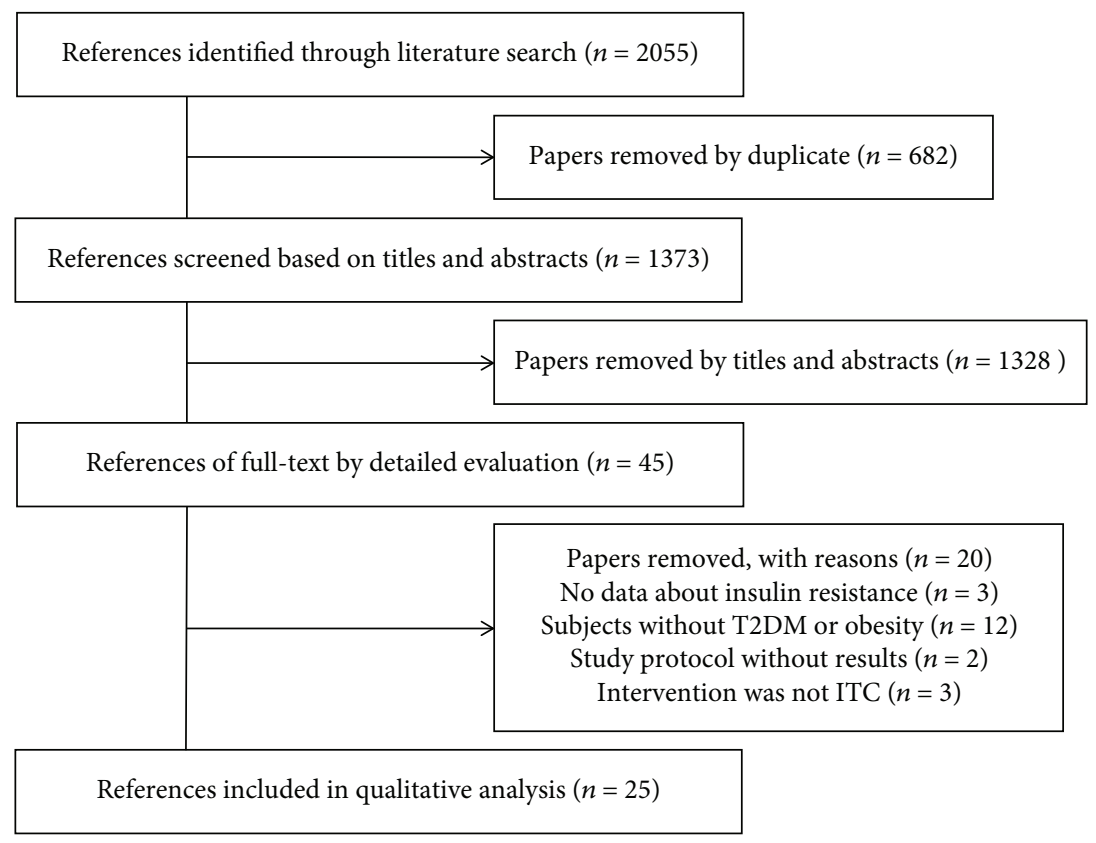

Figure 1: Flow diagram showing study selection.

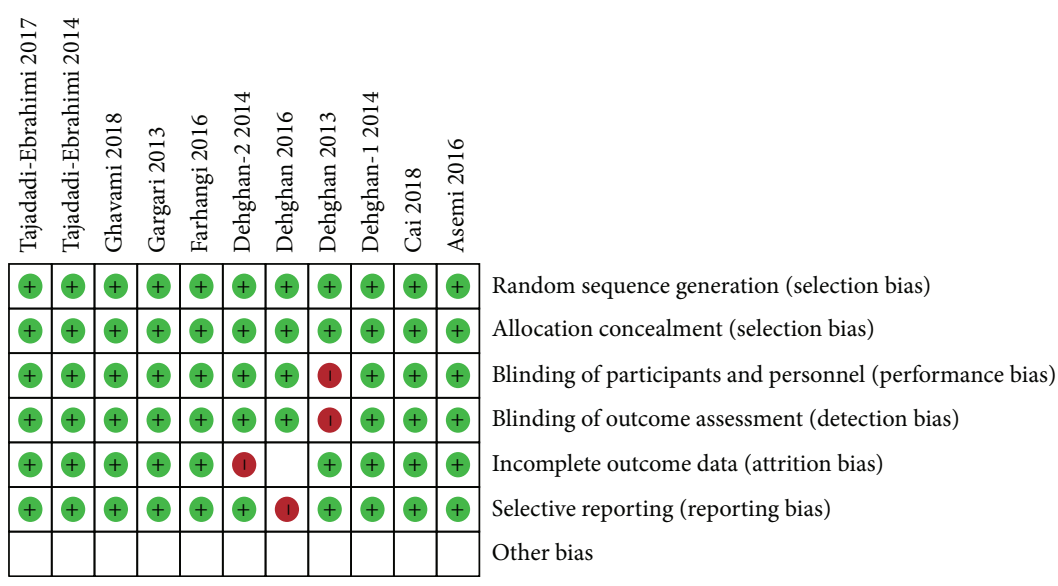

Figure 2: Risk of bias summary for included studies.

which is a most relevant risk factor for T2DM [5]. In patients with T2DM, IR antedates the onset of overt diabetes and may represent a predictive marker for this disease [6, 7]. Obesity in patients with T2DM will aggravate IR [8]. Therefore, body control based on dietary intervention will help to ameliorate IR and improve the efficacy of hypoglycemic medication in T2DM patients [9].

One of the dietary interventions for metabolic disease is the supplementation with inulin-type carbohydrate (ITC), which is a kind of fructan that cannot be digested and absorbed in the small intestine. ITC includes inulin, oligofructose, and fructooligosaccharides, which contain fructose monomers linked by $\beta$ (1-2) bonds [10]. Studies found that ITC could modulate the gut microbiota in animals and humans and promote the proliferation of the beneficial lactic acid-producing Bifidobacteria and Lactobacillus species [11-13]. Gut microbiota is closely related to human health and is also a microorganism that protects the intestines from colonization by exogenous pathogens. In addition, the relationship between gut microbiota and metabolic diseases such as diabetes and obesity has been confirmed by researchers [14]. Moreover, compared with other dietary fibers, ITC exhibits more advantages in glucose tolerance and IR [15]. Preclinical studies have demonstrated that a diet containing inulin can ameliorate IR in diabetic mice [16]. Oligofructose can reduce lymphocytic infiltrate into the pancreatic islets, increase the $\beta$-cell proliferation rate to improve insulin sensitivity and $\beta$-cell function [17]. The review of clinical trials also suggests that ITC supplementation has beneficial effects on metabolic syndrome in individuals with T2DM [18].

Although some studies suggest the advantage of soluble fiber supplementation on IR amelioration in individuals $[19,20]$, there still exists an opposite finding on ITC in T2DM or obese subjects [21-23]. To evaluate the effect of ITC supplementation on IR in T2DM and obese patients, we conducted a systematic review and meta-analysis to choose 


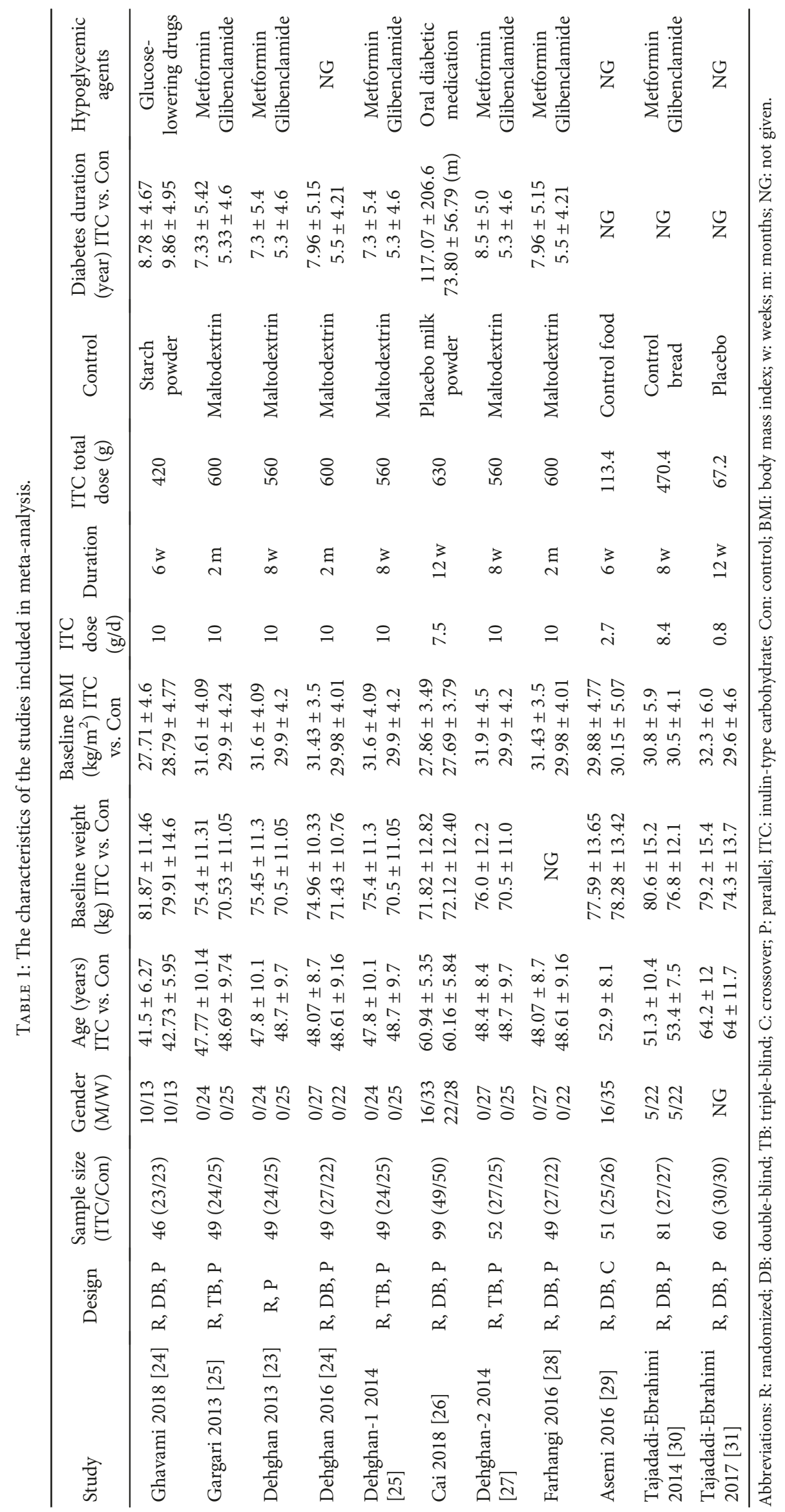




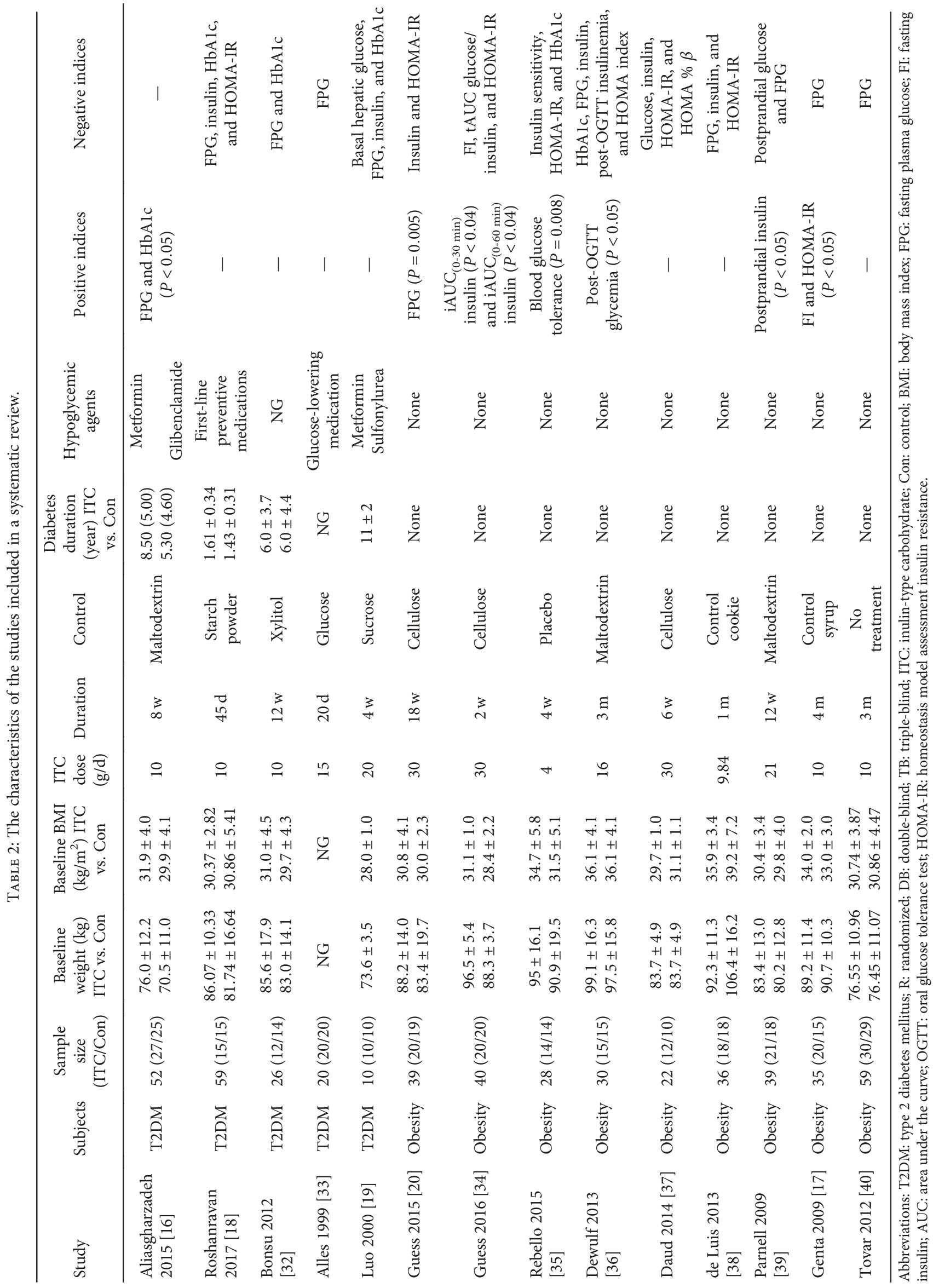




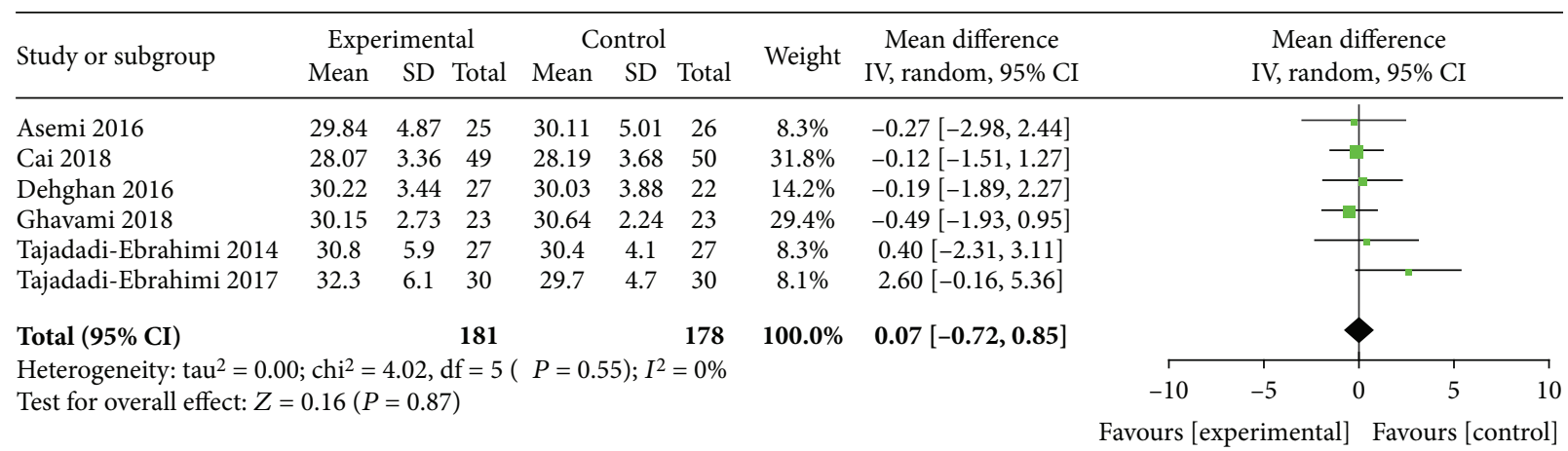

\begin{tabular}{|c|c|c|c|c|c|c|c|c|}
\hline Asemi 2016 & -0.03 & 0.47 & 25 & -0.03 & 0.6 & 26 & $24.2 \%$ & $-0.00[-0.30,0.30]$ \\
\hline Cai 2018 & 0.21 & 0.02 & 49 & 0.5 & 0.02 & 50 & $26.5 \%$ & $-0.29[-0.30,-0.28]$ \\
\hline Dehghan 2016 & -1.21 & 0.41 & 27 & 0.11 & 0.41 & 22 & $25.0 \%$ & $-1.32[-1.55,-1.09]$ \\
\hline Tajadadi-Ebrahimi 2017 & -0.01 & 0.5 & 30 & 0.1 & 0.6 & 30 & $24.4 \%$ & $-0.11[-0.39,0.17]$ \\
\hline Total (95\% CI) & & & 131 & & & 128 & $100.0 \%$ & $-0.43[-0.93,0.06]$ \\
\hline
\end{tabular}

(b)

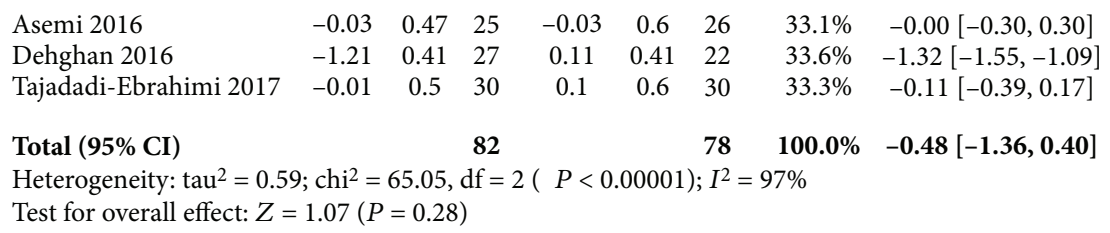

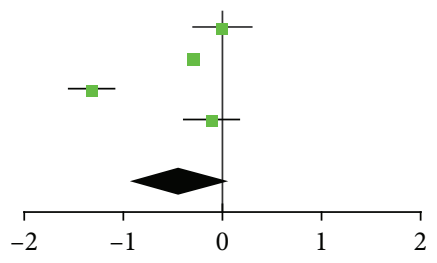

Favours [experimental] Favours [control]

(c)

FIGURE 3: Findings of a meta-analysis of studies with continuation data on improvement in BMI for ITC vs. control groups, in terms of estimated MD and 95\% CI. (a) Postintervention data of BMI, (b) reduction data of BMI, and (c) subgroup analysis for reduction data according to the baseline BMI level.

body mass index (BMI), fasting plasma glucose (FPG), fasting insulin (FI), HbA1c, homeostatic model assessment insulin resistance (HOMA-IR), and the quantitative insulin sensitivity check index (QUICKI) as the indices. The primary outcomes were HOMA-IR and QUICKI.

\section{Methods}

2.1. Literature Search Strategy. This systematic review and meta-analysis was conducted in accordance with guidelines set forth by the Preferred Reporting Items for Systematic Reviews and Meta-Analyses. A literature search was performed on Medline, Embase, ScienceDirect, Web of Science, Cochrane Library, China National Knowledge Infrastructure, and ClinicalTrials.gov to obtain published or grey articles before December 25, 2018. Search terms were inulin in combination with T2DM, obesity, insulin resistance, and insulin sensitivity. The search was performed by two authors independently.

2.2. Inclusion Criteria and Bias Evaluation. The inclusion criteria are the following: (1) the articles described as a randomized clinical trial (RCT) including a parallel and crossover study; (2) studies involved subjects with T2DM and simple obesity (but not merger T2DM); (3) subjects in an experiment group received a dietary ITC intervention compared with the control (placebo or non-ITC supplementation); (4) the outcomes included postintervention and reduction data of $\mathrm{BMI}$, FPG, FI, HbA1c, HOMA-IR, and QUICKI; and (5) the articles were written in English or Chinese. Two reviewers independently assessed the articles based on the titles and abstracts and excluded studies that addressed animal or in vitro experiments, lacked original data, not related to ITC and IR, or duplicated studies, case reports, study protocols, or conference abstracts. The risk of bias was assessed by using the Cochrane Collaboration tool, which included seven specific items: random sequence generation, allocation concealment, blinding of participants and personnel, blinding of outcome assessment, incomplete outcome data, selective reporting, and other biases.

2.3. Definition and Data Extraction. Subjects in the experimental group take ITC and were allowed to treat with hypoglycemic agents during the study course. The forms of ITC (inulin or oligofructose) were either a pure food additive in their daily diet or a mixture of commodities based on ITC. The dose of the mixture was converted into pure ITC. The control group was generally supplemented with a type of digestible carbohydrate which cannot be fermented. The following important items were extracted from each included 


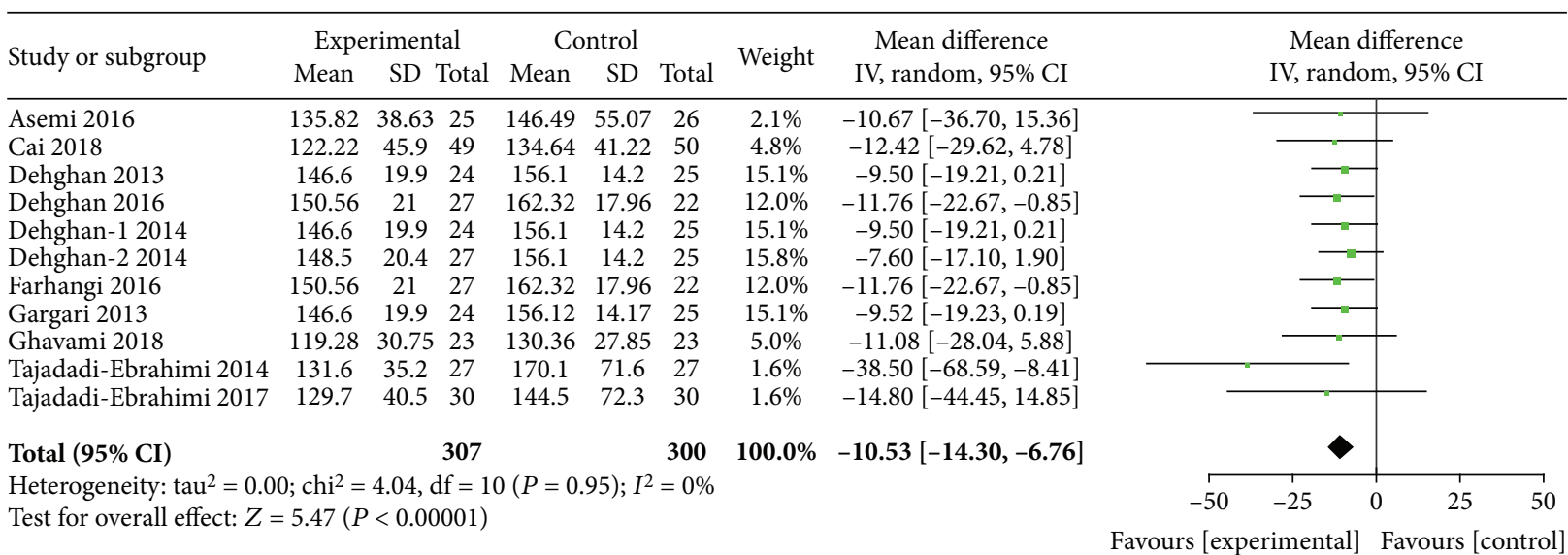

(a)

\begin{tabular}{|c|c|c|c|c|c|c|}
\hline Asemi 2016 & -1.61 & 43.65 & 25 & 3.67 & 35.14 & 26 \\
\hline Cai 2018 & -29.16 & 2.304 & 49 & -11.88 & 2.106 & 50 \\
\hline Dehghan 2016 & -14.25 & 2.37 & 27 & 2.4 & 2.65 & 22 \\
\hline Dehghan-1 2014 & -15.1 & 6.173 & 24 & -1.7 & 2.551 & 25 \\
\hline Dehghan-2 2014 & -18.3 & 3.676 & 27 & -1.7 & 2.551 & 25 \\
\hline Tajadadi-Ebrahimi 2014 & -11.3 & 55.5 & 27 & 0.2 & 72.4 & 27 \\
\hline Tajadadi-Ebrahimi 2017 & -19.6 & 75.6 & 30 & 19.2 & 66.9 & 30 \\
\hline Total (95\% CI) & & & 209 & & & 205 \\
\hline
\end{tabular}

Test for overall effect: $Z=26.67(P<0.00001)$

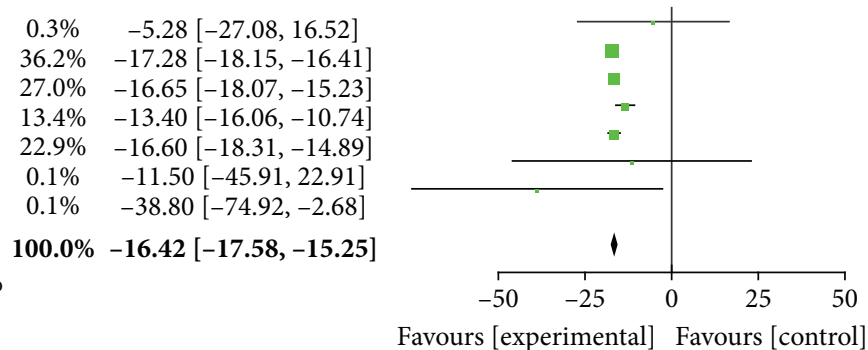

(b)

FIGURE 4: Findings of a meta-analysis of studies with continuation data on improvement in FPG for ITC vs. control groups, with estimated MD and 95\% CI. (a) Postintervention data of FPG. (b) Reduction data of FPG.

RCT: study design, subjects, sample size, baseline BMI, ITC dose and duration, and outcomes. An effort was made to email article authors to obtain data which are not shown in the published paper. All data were independently extracted by C.L. Gao and M.Y. Rao and confirmed by L. Xu and L. Jiang. Disagreements about eligibility and the extracted items were resolved by discussion between all authors, and the corresponding author $(\mathrm{Y} . \mathrm{Xu})$ ruled on disagreements.

2.4. Statistical Analysis. All analyses were carried out using the Review Manager software, version 5.0 (Cochrane, Copenhagen, Denmark). The FPG and FI units in all the studies were converted to be the same, and then the data were pooled to calculate the mean difference (MD) and $95 \%$ confidence interval (CI). Review Manager generated forest plots of the pooled MDs with 95\% CIs for all outcomes. Allowing for heterogeneity between the studies, the data were pooled using a random effects model to facilitate generalizability of results. Statistical heterogeneity was assessed using $Q$ tests and the $I^{2}$ statistic. Subgroup analysis was carried out according to the clinical characteristics of the subjects to eliminate heterogeneity.

\section{Results}

Our search yielded 2055 studies for an initial review. After screening titles and abstracts, 45 full-text articles were reviewed. 20 of these articles did not meet inclusion criteria, and the remaining 11 articles which comprised 634 T2DM patients were finally included in this meta-analysis [24-34]
(Figure 1). In general, the included studies can be considered to have a lower risk of bias (Figure 2). Another 14 studies were systematically evaluated because the data cannot be pooled, including T2DM and obese subjects [19-23, 3543]. The basic characteristics for all selected studies were shown in Tables 1 and 2.

3.1. Trial Characteristics. Eleven studies used for metaanalysis were designed to be random and double-blind or triple-blind. Only the Dehghan et al. study [26] did not report whether the study was blind. The Asemi et al. study [32] was crossover-designed, while the rest were paralleldesigned. All of the studies involved patients with T2DM; six studies of which were female subjects only. The average baseline BMI of the subjects ranged from 27.69 to $31.9 \mathrm{~kg} / \mathrm{m}^{2}$, and the BMI in the Ghavami et al. and Cai et al. studies $[24,29]$ was lower than $28 \mathrm{~kg} / \mathrm{m}^{2}$. The daily dose of ITC ranged from 2.7 to $10 \mathrm{~g}$, and the duration of ITC ranged from 6 to 12 weeks. The ITC dose in the Tajabadi-Ebrahimi et al. study [34] was lower than that in the other studies. Therefore, the subgroup analyses were performed based on baseline BMI and ITC daily dosage.

3.2. Effects of ITC Supplementation on Posttreatment BMI. We analyzed postintervention BMI data of T2DM patients. Our results did not show that ITC supplementation decreases the BMI in whole individuals $\left(I^{2}=0 \% ; P=0.87\right.$ ) (Figure 3(a)). In addition, the reduction data of the BMI after ITC supplementation also showed that it was not significantly lower 


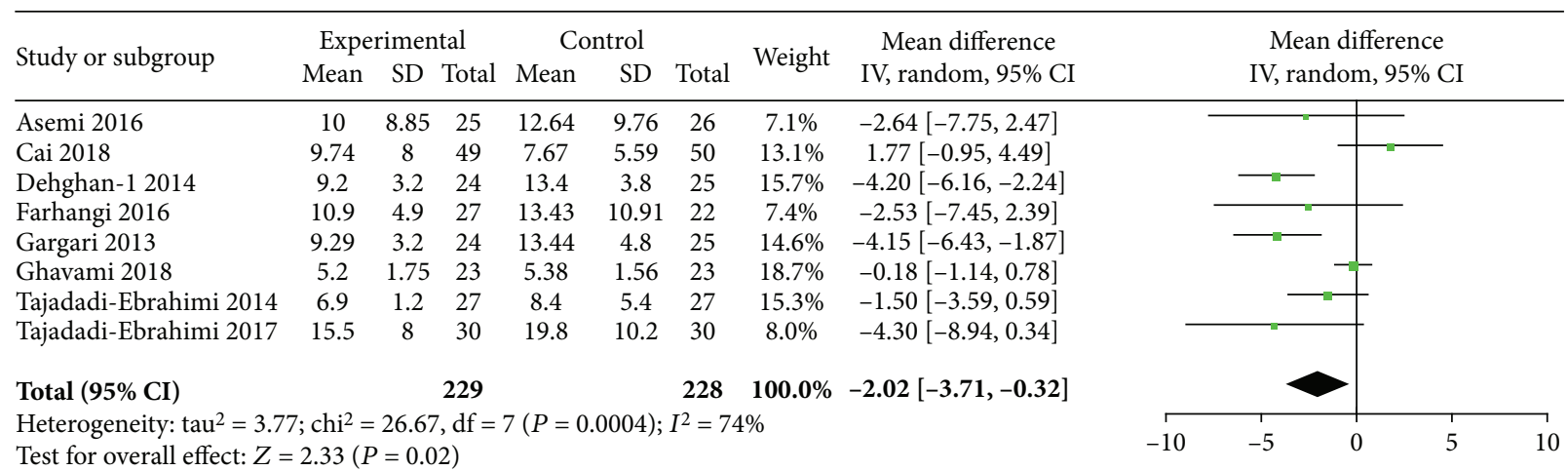

(a)

\begin{tabular}{|c|c|c|c|c|c|c|c|c|}
\hline Asemi 2016 & 10 & 8.85 & 25 & 12.64 & 9.76 & 26 & $4.7 \%$ & $-2.64[-7.75,2.47]$ \\
\hline Dehghan-1 2014 & 9.2 & 3.2 & 24 & 13.4 & 3.8 & 25 & $32.1 \%$ & $-4.20[-6.16,-2.24]$ \\
\hline Farhangi 2016 & 10.9 & 4.9 & 27 & 13.43 & 10.91 & 22 & $5.1 \%$ & $-2.53[-7.45,2.39]$ \\
\hline Gargari 2013 & 9.29 & 3.2 & 24 & 13.44 & 4.8 & 25 & $23.9 \%$ & $-4.15[-6.43,-1.87]$ \\
\hline Tajadadi-Ebrahimi 2014 & 6.9 & 1.2 & 27 & 8.4 & 5.4 & 27 & $28.4 \%$ & $-1.50[-3.59,0.59]$ \\
\hline Tajadadi-Ebrahimi 2017 & 15.5 & 8 & 30 & 19.8 & 10.2 & 30 & $5.8 \%$ & $-4.30[-8.94,0.34]$ \\
\hline Total $(95 \% \mathrm{CI})$ & & & 157 & & & 155 & $100.0 \%$ & $-3.27[-4.38,-2.15]$ \\
\hline
\end{tabular}

Favours [experimental] Favours [control]

Heterogeneity: $\operatorname{tau}^{2}=0.00 ; \mathrm{chi}^{2}=4.53, \mathrm{df}=5(P=0.48) ; I^{2}=0 \%$

Test for overall effect: $Z=5.76(P<0.00001)$

(b)

\begin{tabular}{lccccccc} 
Asemi 2016 & -1 & 7.9 & 25 & 3.68 & 6.91 & 26 & $17.2 \%$ \\
Cai 2018 & 0.91 & 0.296 & 49 & 0.32 & 0.286 & 50 & $22.0 \%$ \\
Dehghan-1 2014 & -4.8 & 1.02 & 24 & 0.2 & 0.153 & 25 & $22.0 \%$ \\
Tajadadi-Ebrahimi 2014 & -3.2 & 5.4 & 27 & 0.6 & 4.7 & 27 & $19.6 \%$ \\
Tajadadi-Ebrahimi 2017 & -0.7 & 5.1 & 30 & 3.3 & 6.3 & 30 & $19.3 \%$ \\
& \multicolumn{7}{c}{$\mathbf{1 5 5}$} \\
Total (95\% CI) & \\
Heterogeneity: tau ${ }^{2}=15.23$; chi $^{2}=676.74, \mathrm{df}=4(P<0.00001) ; I^{2}=99 \%$ & $\mathbf{1 0 0 . 0 \%}$ \\
Test for overall effect: $Z=1.79(P=0.07)$
\end{tabular}

(c)
$1.0 \%-4.68[-8.76,-0.60]$

$94.9 \%-5.00[-5.41,-4.59]$

$2.2 \%-3.80[-6.50,-1.10]$

$1.9 \%-4.00[-6.90,-1.10]$

$100.0 \%-4.95[-5.35,-4.55]$
$-4.68[-8.76,-0.60]$

$-5.00[-5.41,-4.59]$

$-3.80[-6.50,-1.10]$

$-3.29[-6.88,-0.30]$

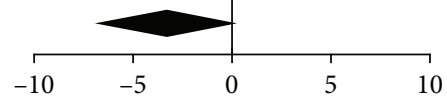

Favours [experimental] Favours [control]

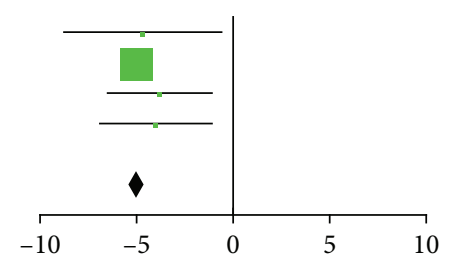

Favours [experimental] Favours [control]

(d)

FIGURE 5: Findings of a meta-analysis of studies with continuation data on improvement in FI for ITC vs. control groups, with estimated MD and 95\% CI. (a) Postintervention data of FI, (c) reduction data of FI, and (b, d) subgroup analysis according to the baseline BMI level.

than that in the control group (MD, -0.43; 95\% CI, - $0.93-$ $\left.0.06 ; I^{2}=96 \% ; P=0.08\right)$. Subgroup analysis excluding studies of the baseline BMI less than $28 \mathrm{~kg} / \mathrm{m}^{2}$ also found no difference between the ITC and control groups (MD, $-0.48 ; 95 \%$ CI, $-1.36-0.40 ; I^{2}=97 \% ; P=0.28$ ) (Figures $3(b)$ and $3(c)$ ).

3.3. Glucose, Insulin, and HbAlc Reduction by ITC Intervention. A total of 11 studies analyzed FPG for all subjects, and 8 articles studied FI and HbA1c. The FPG levels of all subjects were significantly lower in the ITC intervention group $\left(I^{2}=0 \% ; P<0.00001\right)$, and the reduction data of the
FPG levels before and after the intervention were also more significant in the ITC group (MD, -16.42; 95\% CI, -17.58 to $-15.25 ; I^{2}=41 \% ; P<0.00001$ ) (Figures $4(\mathrm{a})$ and $4(\mathrm{~b})$ ). After ITC consumption, the FI level was lower than that in the control group, but there was significant heterogeneity $\left(I^{2}=74 \%\right.$; $P=0.02$ ) (Figure 5(a)). Subgroup analysis based on the baseline BMI could eliminate the heterogeneity $\left(I^{2}=0 \% ; P<\right.$ 0.00001) (Figure 5(b)). The reduction data of FI after the ITC intervention were not significant compared with the control group (MD, -3.29; 95\% CI, -6.88-0.3; $I^{2}=99 \% ; P=$ $0.07)$, but subgroup analysis found that the ITC group has 


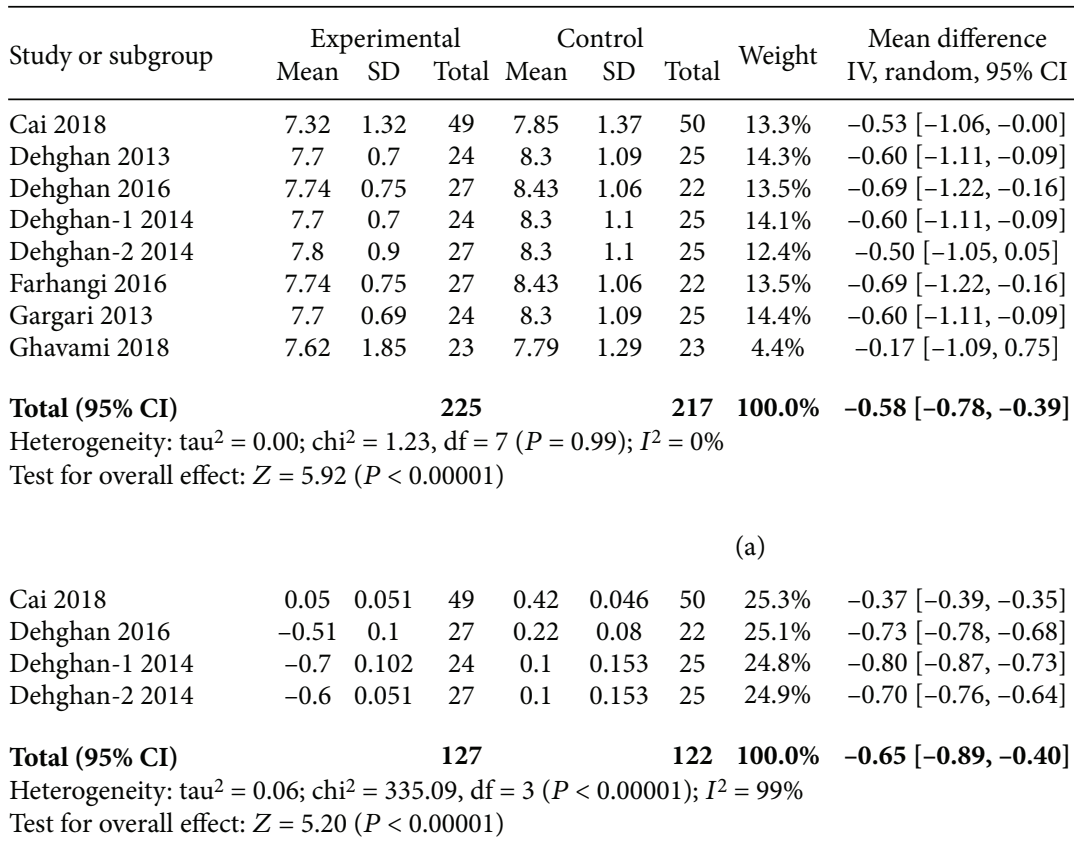

(b)

\begin{tabular}{|c|c|c|c|c|c|c|c|c|}
\hline Dehghan 2016 & -0.51 & 0.1 & 27 & 0.22 & 0.08 & 22 & $39.3 \%$ & $-0.73[-0.78,-0.68]$ \\
\hline Dehghan-1 2014 & -0.7 & 0.102 & 24 & 0.1 & 0.153 & 25 & $28.1 \%$ & $-0.80[-0.87,-0.73]$ \\
\hline Dehghan-2 2014 & -0.6 & 0.051 & 27 & 0.1 & 0.153 & 25 & $32.5 \%$ & $-0.70[-0.76,-0.64]$ \\
\hline Total $(95 \% \mathrm{CI})$ & & & 78 & & & 72 & $100.0 \%$ & $-0.74[-0.79,-0.69]$ \\
\hline
\end{tabular}

(c)

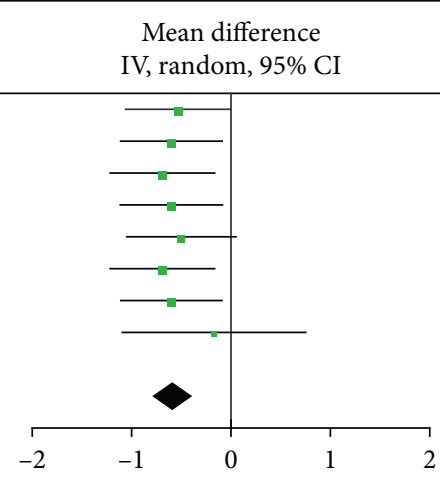

Favours [experimental] Favours [control]

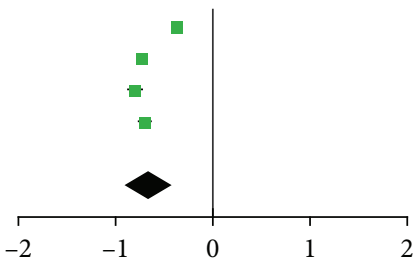

Favours [experimental] Favours [control]

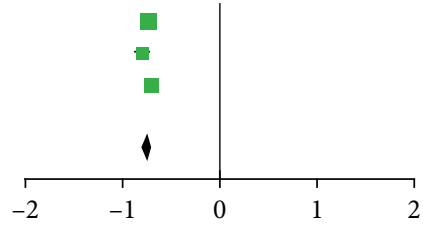

Favours [experimental] Favours [control]

FIGURE 6: Findings of a meta-analysis of studies with continuation data on amelioration in HbA1c for ITC vs. control groups, with estimated MD and 95\% CI. (a) Postintervention data of HbA1c, (b) reduction data of HbAlc, and (c) subgroup analysis for reduction data according to the baseline BMI level.

a significant FI reduction $\left(I^{2}=0 \% ; P<0.00001\right)$ (Figures $5(\mathrm{c})$ and $5(\mathrm{~d}))$. The postintervention $\mathrm{HbA1c}$ level and change data of HbA1c consistently showed that the ITC group had absolute advantages, but the change data had heterogeneity $\mathrm{MD}$, $-0.58 \%$; $95 \%$ CI, $-0.78 \%$ to $-0.39 \% ; I^{2}=0 \% ; P<0.00001$; and MD, $-0.65 \%$; $95 \%$ CI, $-0.89 \%$ to $-0.4 \%$; $I^{2}=99 \%$; $P<0.00001$, respectively). Subgroup analysis based on the BMI could eliminate partial heterogeneity $\left(I^{2}=53 \% ; \quad P<0.00001\right)$ (Figures 6(a)-6(c)).

3.4. Ameliorated Effect of ITC Intervention on IR. The fasting IR index mainly includes the HOMA-IR and the QUICKI. ITC intervention significantly ameliorated HOMA-IR, either the postintervention HOMA-IR level or the reduction data of HOMA-IR (MD, $-0.99 ; 95 \% \mathrm{CI},-1.76$ to $-0.21, I^{2}=75 \%$, $P=0.01$; and MD, $-0.99 ; 95 \% \mathrm{CI},-1.62$ to $-0.35, I^{2}=42 \%$, $P=0.002$, respectively). Heterogeneity can be eliminated by subgroup analysis according to the baseline BMI $\left(I^{2}=0 \%\right.$; $P<0.00001$ ) (Figures $7(\mathrm{a})-7(\mathrm{c})$ ). Only 3 articles studied QUICKI. Meta-analysis found that there was no statistical difference between the ITC and control groups on postinter- vention data of QUICKI (MD, 0.01; 95\% CI, 0.00-0.03; $I^{2}=$ $70 \% ; P=0.13)$. According to the dose of ITC intake, the subgroup analysis showed the statistically significant difference after the study of low-dose ITC intake was excluded (MD, 0.02; 95\% CI, 0.01-0.03; $I^{2}=0 \%$; $\left.<<0.0001\right)$. However, there was no statistical difference in QUICKI reduction between the two groups (MD, 0.00; 95\% CI, $-0.01-0.02 ; I^{2}=74 \%$; $P=0.64)$ and so did subgroup analysis $\left(I^{2}=49 \% ; P=0.79\right)$ (Figures 8(a)-8(d)).

3.5. Systematic Review of ITC Intervention on the Glycometabolism and Homeostasis Model. Data from 14 other studies, including T2DM (5 studies) and simple obese (9 studies) patients, could not be pooled (Table 2). Except Alles et al.' study [36] which does not give the baseline BMI data, all subjects' average baseline BMI was greater than $28 \mathrm{~kg} / \mathrm{m}^{2}$. In the ITC intervention group, the daily dose ranged from 4 to $30 \mathrm{~g}$ and the duration ranged from 2 weeks to 4 months. It was found that the conclusions about blood glucose, insulin, HbA1c, and HOMA-IR were complicated. Only Aliasgharzadeh et al. [19] found that ITC intervention could significantly decrease FPG and HbA1c in T2DM 


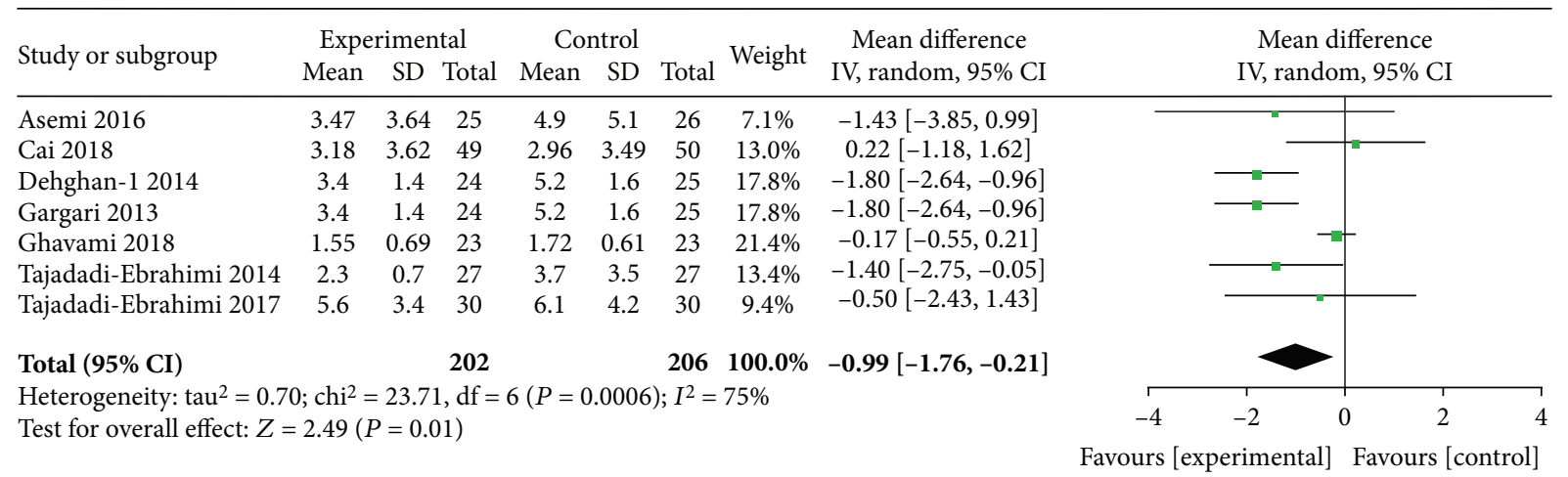

(a)

\begin{tabular}{|c|c|c|c|c|c|c|c|c|}
\hline Asemi 2016 & 3.47 & 3.64 & 25 & 4.9 & 5.1 & 26 & $4.5 \%$ & $-1.43[-3.85,0.99]$ \\
\hline Dehghan-1 2014 & 3.4 & 1.4 & 24 & 5.2 & 1.6 & 25 & $37.0 \%$ & $-1.80[-2.64,-0.96]$ \\
\hline Gargari 2013 & 3.4 & 1.4 & 24 & 5.2 & 1.6 & 25 & $37.0 \%$ & $-1.80[-2.64,-0.96]$ \\
\hline Tajadadi-Ebrahimi 2014 & 2.3 & 0.7 & 27 & 3.7 & 3.5 & 27 & $14.5 \%$ & $-1.40[-2.75,-0.05]$ \\
\hline Tajadadi-Ebrahimi 2017 & 5.6 & 3.4 & 30 & 6.1 & 4.2 & 30 & $7.0 \%$ & $-0.50[-2.43,1.43]$ \\
\hline Total $(95 \%$ CI $)$ & & & 130 & & & 133 & $100.0 \%$ & $-1.63[-2.15,-1.12]$ \\
\hline
\end{tabular}

(b)

\begin{tabular}{|c|c|c|c|c|c|c|c|c|}
\hline Asemi 2016 & -0.73 & 3.96 & 25 & 1.82 & 4.09 & 26 & $7.2 \%$ & $-2.55[-4.76,-0.34]$ \\
\hline Cai 2018 & -0.32 & 0.163 & 49 & 0.33 & 0.133 & 50 & $57.2 \%$ & $-0.65[-0.71,-0.59]$ \\
\hline Tajadadi-Ebrahimi 2014 & -1.5 & 2.7 & 27 & 0.4 & 3.5 & 27 & $11.6 \%$ & $-1.90[-3.57,-0.23]$ \\
\hline Tajadadi-Ebrahimi 2017 & 0.01 & 1.8 & 30 & 0.9 & 2.1 & 30 & $24.0 \%$ & $-0.89[-1.88,0.10]$ \\
\hline Total $(95 \%$ CI) & & & 131 & & & 133 & $100.0 \%$ & $-0.99[-1.62,-0.35$ \\
\hline
\end{tabular}

(c)

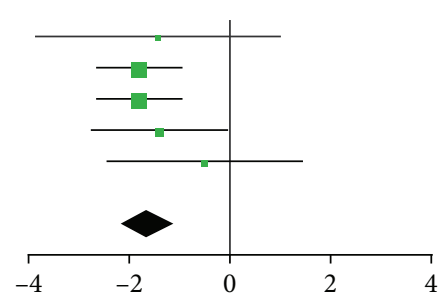

Favours [experimental] Favours [control]

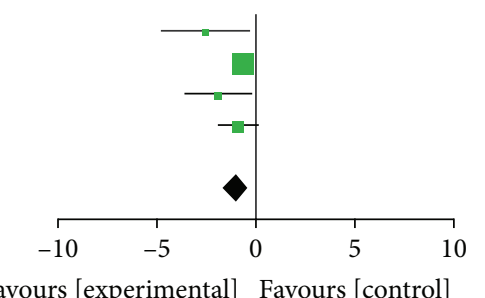

Favours [experimental] Favours [control]

FIgURE 7: Findings of a meta-analysis of studies with continuation data on amelioration in HOMA-IR for ITC vs. control groups, with estimated MD and 95\% CI. (a) Postintervention data of HOMA-IR, (b) subgroup analysis for postintervention data according to the baseline BMI level, and (c) reduction data of HOMA-IR.

patients $(P<0.05)$; none of the other four studies reached a positive conclusion. Moreover, 9 studies on obese patients found that the results of blood glucose, insulin, and HbAlc were controversial. However, only Genta et al.'s [20] study on HOMA-IR concluded that ITC intervention was statistically significant, while the other six studies did not.

\section{Discussion}

IR is not only the central link and treatment target of T2DM but also one of the mechanisms of other diseases secondary to T2DM. Studies have found that IR and hyperglycemia can increase the risk of adverse cardiovascular events [44] and suggested a link between IR in T2DM patients and cognitive dysfunction [45] and Parkinson's disease [46]. The main index to evaluate IR is hyperinsulinemic-euglycemic clamp, HOMA-IR, and QUICKI. In recent years, many RCTs about the effect of ITC on the amelioration of blood glucose and IR have been reported $[19,20]$ and some systematic reviews on the effect of ITC on blood lipid, triacylglycerols, and chronic constipation have been carried out $[10,47,48]$.
However, the meta-analysis of ITC-ameliorated IR has not been conducted. In the present study, we made this review involving 25 RCTs of parallel or crossover; to our knowledge, this was the first systematic analysis to evaluate the role of ITC supplementation in ameliorating IR in T2DM and obese patients.

We found the explicit effect of ITC supplementation on glycometabolism and HOMA-IR amelioration in T2DM with obesity. Postintervention and reduction outcomes of FPG, HbAlc, and HOMA-IR were significantly ameliorated after ITC supplementation in meta-analysis. Insulin secretion decreased significantly after inulin intake, but there was no significant difference between the reduction data of two groups $\left(I^{2}=99 \%, P=0.07\right)$. With the heterogeneity, we noted that the baseline BMI may affect outcomes. In the study where the baseline BMI was greater than the $28 \mathrm{~kg} / \mathrm{m}^{2}$, the subgroup analysis found that the difference was statistical and the heterogeneity was eliminated. The data of QUICKI, another index of IR, was collected in three studies and showed that there was no statistical difference between the two groups. Based on the characteristics of these 


\begin{tabular}{|c|c|c|c|c|c|c|c|c|c|c|c|c|}
\hline \multirow{2}{*}{ Study or subgroup } & \multicolumn{3}{|c|}{ Experimental } & \multicolumn{3}{|c|}{ Control } & \multirow{2}{*}{ Weight } & \multirow{2}{*}{$\begin{array}{l}\text { Mean difference } \\
\text { IV, random, 95\% CI }\end{array}$} & \multirow{2}{*}{\multicolumn{4}{|c|}{$\begin{array}{l}\text { Mean difference } \\
\text { IV, random, 95\% CI }\end{array}$}} \\
\hline & Mean & $\mathrm{SD}$ & Total & Mean & $\mathrm{SD}$ & Total & & & & & & \\
\hline Asemi 2016 & 0.34 & 0.06 & 25 & 0.32 & 0.04 & 26 & $19.0 \%$ & $0.02[-0.01,0.05]$ & \multicolumn{4}{|c|}{ t- } \\
\hline Tajadadi-Ebrahimi 2014 & 0.34 & 0.02 & 27 & 0.32 & 0.02 & 27 & $41.0 \%$ & $0.02[0.01,0.03]$ & \multicolumn{4}{|c|}{ 늘 } \\
\hline Tajadadi-Ebrahimi 2017 & 0.3 & 0.02 & 20 & 0.3 & 0.02 & 30 & $40.0 \%$ & $0.00[-0.01,0.01]$ & \multicolumn{4}{|c|}{$\frac{1}{1}$} \\
\hline Total $(95 \%$ CI $)$ & & & 72 & & & 83 & $100.0 \%$ & $0.01[-0.00,-0.03]$ & & & & \\
\hline \multicolumn{7}{|c|}{$\begin{array}{l}\text { Heterogeneity: } \operatorname{tau}^{2}=0.00 ; \mathrm{chi}^{2}=6.75, \mathrm{df}=2(P=0.03) ; I^{2}=70 \% \\
\text { Test for overall effect: } Z=1.52(P=0.13)\end{array}$} & & & -0.2 & -0.1 & 0.1 & 0.2 \\
\hline
\end{tabular}

(a)

$\begin{array}{lcccccccc}\text { Asemi 2016 } & 0.34 & 0.06 & 25 & 0.32 & 0.04 & 26 & 12.6 \% & 0.02[-0.01,0.05] \\ \text { Tajadadi-Ebrahimi 2014 } & 0.34 & 0.02 & 27 & 0.32 & 0.02 & 27 & 87.4 \% & 0.02[0.01,0.03] \\ & & & & & & & & \\ \text { Total (95\% CI) } & & & \mathbf{5 2} & & & \mathbf{5 3} & \mathbf{1 0 0 . 0 \%} & \mathbf{0 . 0 2}[\mathbf{0 . 0 1}, \mathbf{0 . 0 3}]\end{array}$

Heterogeneity: $\operatorname{tau}^{2}=0.00 ; \mathrm{chi}^{2}=0.00, \mathrm{df}=1(P=1.00) ; I^{2}=0 \%$

Test for overall effect: $Z=3.93(P<0.0001)$

(b)

$\begin{array}{lcccccccc}\text { Asemi 2016 } & 0.001 & 0.06 & 25 & -0.01 & 0.03 & 26 & 21.2 \% & 0.01[-0.02,0.04] \\ \text { Tajadadi-Ebrahimi 2014 } & -0.013 & 0.02 & 27 & -0.003 & 0.03 & 27 & 35.8 \% & -0.01[-0.02,0.00] \\ \text { Tajadadi-Ebrahimi 2017 } & 0.002 & 0.01 & 30 & -0.01 & 0.02 & 30 & 42.9 \% & 0.01[0.00,0.02] \\ & & & & & & & & \\ \text { Total (95\% CI) } & & & \mathbf{8 2} & & & \mathbf{8 3} & \mathbf{1 0 0 . 0 \%} & \mathbf{0 . 0 0 [ - 0 . 0 1 , 0 . 0 2}]\end{array}$

Heterogeneity: $\operatorname{tau}^{2}=0.00 ; \mathrm{chi}^{2}=7.58, \mathrm{df}=2(P=0.02) ; I^{2}=74 \%$

Test for overall effect: $Z=0.47(P=0.64)$

Favours [control] Favours [experimental]

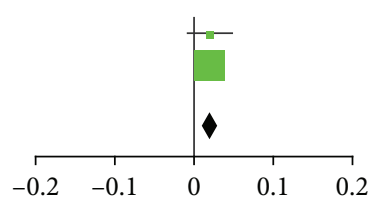

Favours [control] Favours [experimental]

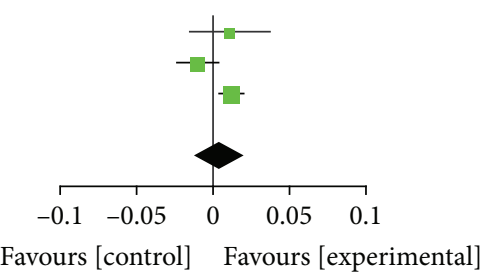

(c)

\begin{tabular}{|c|c|c|c|c|c|}
\hline Asemi 2016 & 0.001 & 0.06 & 25 & -0.01 & 0.03 \\
\hline Tajadadi-Ebrahimi 2014 & -0.013 & 0.02 & 27 & -0.003 & 0.03 \\
\hline Total (95\% CI) & & & 52 & & \\
\hline
\end{tabular}

$35.2 \% \quad 0.01[-0.02,0.04]$

$64.8 \%-0.01[-0.02,0.00]$

$100.0 \%-0.00[-0.02,0.02]$

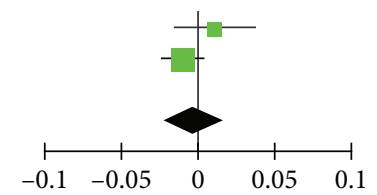

Favours [control] Favours [experimental]

(d)

FIGURE 8: Findings of a meta-analysis of studies with continuation data on improvement in QUICKI for ITC vs. control groups, with estimated MD and 95\% CI. (a) Postintervention data of QUICKI, (c) reduction data of QUICKI, and (b, d) subgroup analysis according to the baseline BMI level.

studies, we speculated that the outcome may be related to the daily intakes of ITC. However, because the number of studies is too small, the conclusion cannot be generalized. As data could not be pooled, we systematically reviewed nine studies of obese people. The indicator of IR is the HOMA-IR; six studies concluded that ITC was ineffective.

As well known, obesity is closely associated with type 2 diabetes. However, this study found that ITC consumption had a controversial effect on IR in simple obesity but a significant result in T2DM, especially in T2DM with obesity. ITC could not be digested and absorbed in the small intestine but could be fermented by the microbial flora in the large bowel $[49,50]$. In addition, ITC can modulate the composition of gut microbiota and increase the formation of short-chain fatty acids (SCFAs) in the process of intestinal fermentation $[51,52]$. SCFAs had been shown to increase insulin sensitivity, improve glucose tolerance, and reduce $\beta$ cell apoptosis in obese and diabetic animals $[53,54]$ and could also stimulate intestinal gluconeogenesis [55]. Moreover, several mechanisms can explain the beneficial effects of a diet containing inulin on metabolism. It might be attrib- uted to their impact on gene expression [56] and modulation of the intestinal microbiota, SCFAs, and hormone axis, especially with regard to increased promotion of the hormone glucagon-like peptide-1 [57]. In addition, changes in the levels of gut hormones like peptide YY [58] and activation of the lipopolysaccharide Toll-like receptor-2 were also mechanisms [59]. The effect of ITC on IR was related to the increase of specific intestinal flora [60]; maybe, ITC had different effects on the intestinal microbes in simple obesity and T2DM.

There were some limitations in this study. First, the amount of studies included in this meta-analysis was small and some studies have small sample size, so the random error existed and bias of results may occur. Second, the oral glucose tolerance test is recommended to assess IR in clinical practice commonly [61], but none of the studies included in this meta-analysis conducted OGTT tests, so we did not obtain $2 \mathrm{~h}$ postprandial blood glucose data. Third, subgroup analysis on the baseline BMI or inulin intake dosage in T2DM may have an unpredictable bias and the BMI was an independent factor required for Cox model analysis in multiple clinical 
trials. It suggested that much clinic trials will be needed to clarify the impact of ITC supplementation on the prevention and treatment of metabolic diseases. Finally, this metaanalysis did not have strict exclusion criteria in order to include all related studies as much as possible. We only excluded the studies that have normal people subjects. However, the studies included had some common exclusion criteria, such as subjects had a history of gastrointestinal, pancreatic, or cardiovascular disease, renal, thyroid, or liver disturbance, being pregnant or lactating, consuming pre- or probiotic products, antibiotics, antidiarrheal, anti-inflammatory, or laxative drugs, or if the subjects had a daily fiber intake $>30 \mathrm{~g}$. In addition, the differences in these studies, such as baseline BMI levels in subjects, duration of diabetes, and dose and duration of ITC, were subgroup analyzed to determine the significance of these factors. Therefore, we think that the above limitations did not influence our conclusion that ITC supplementation can ameliorate IR in T2DM, especially in patients with obesity.

\section{Conclusions}

This meta-analysis indicates that the supplementation of ITC is efficacious in glycemic control and IR amelioration in T2DM, especially in obese T2DM patients. However, it is controversial in obese patients. Meanwhile, more randomized, double-blind, and large-sample-sized trials of ITC for T2DM and simple obese are needed in the future to validate or revise the result of this work.

\section{Disclosure}

Chenlin Gao is a co-first author.

\section{Conflicts of Interest}

The authors declare that they have no conflict of interest.

\section{Acknowledgments}

The authors gratefully acknowledge BioMed Proofreading for assistance with English expression. This work was supported by the Educational Fund of Sichuan Province (17CZ0041).

\section{References}

[1] R. A. DeFronzo, E. Ferrannini, L. Groop et al., "Type 2 diabetes mellitus," Nature Reviews Disease Primers, vol. 1, no. 1, article 15019, 2015.

[2] S. E. Kahn, "The relative contributions of insulin resistance and beta-cell dysfunction to the pathophysiology of type 2 diabetes," Diabetologia, vol. 46, no. 1, pp. 3-19, 2003.

[3] N. H. Cho, J. E. Shaw, S. Karuranga et al., "IDF Diabetes Atlas: global estimates of diabetes prevalence for 2017 and projections for 2045," Diabetes Research and Clinical Practice, vol. 138, pp. 271-281, 2018.

[4] L. T. F. Cheung, R. S. M. Chan, G. T. C. Ko, E. S. H. Lau, F. C. C. Chow, and A. P. S. Kong, "Diet quality is inversely associ- ated with obesity in Chinese adults with type 2 diabetes," Nutrition Journal, vol. 17, no. 1, p. 63, 2018.

[5] W. C. Knowler, E. Barrett-Connor, S. E. Fowler et al., "Reduction in the incidence of type 2 diabetes with lifestyle intervention or metformin," The New England Journal of Medicine, vol. 346, no. 6, pp. 393-403, 2002.

[6] A. Brunetti, L. Brunetti, D. Foti, D. Accili, and I. D. Goldfine, "Human diabetes associated with defects in nuclear regulatory proteins for the insulin receptor gene," Journal of Clinical Investigation, vol. 97, no. 1, pp. 258-262, 1996.

[7] E. Chiefari, F. Paonessa, S. Iiritano et al., "The cAMPHMGA1-RBP4 system: a novel biochemical pathway for modulating glucose homeostasis," BMC Biology, vol. 7, no. 1, p. 24, 2009.

[8] S. E. Kahn, R. L. Hull, and K. M. Utzschneider, "Mechanisms linking obesity to insulin resistance and type 2 diabetes," Nature, vol. 444, no. 7121, pp. 840-846, 2006.

[9] D. Houghton, T. Hardy, C. Stewart et al., "Systematic review assessing the effectiveness of dietary intervention on gut microbiota in adults with type 2 diabetes," Diabetologia, vol. 61, no. 8, pp. 1700-1711, 2018.

[10] N. Gupta, A. K. Jangid, D. Pooja, and H. Kulhari, "Inulin: a novel and stretchy polysaccharide tool for biomedical and nutritional applications," International Journal of Biological Macromolecules, vol. 132, pp. 852-863, 2019.

[11] N. M. Delzenne, A. M. Neyrinck, F. Backhed, and P. D. Cani, "Targeting gut microbiota in obesity: effects of prebiotics and probiotics," Nature Reviews Endocrinology, vol. 7, no. 11, pp. 639-646, 2011.

[12] N. M. Delzenne, A. M. Neyrinck, and P. D. Cani, "Modulation of the gut microbiota by nutrients with prebiotic properties: consequences for host health in the context of obesity and metabolic syndrome," Microbial Cell Factories, vol. 10, article S10, Supplement 1, 2011.

[13] C. Ramirez-Farias, K. Slezak, Z. Fuller, A. Duncan, G. Holtrop, and P. Louis, "Effect of inulin on the human gut microbiota: stimulation of Bifidobacterium adolescentis and Faecalibacterium prausnitzii," British Journal of Nutrition, vol. 101, no. 4, pp. 541-550, 2009.

[14] E. E. Canfora, R. C. R. Meex, K. Venema, and E. E. Blaak, "Gut microbial metabolites in obesity, NAFLD and T2DM," Nature Reviews. Endocrinology, vol. 15, no. 5, pp. 261-273, 2019.

[15] L. F. de Cossío, C. Fourrier, J. Sauvant et al., "Impact of prebiotics on metabolic and behavioral alterations in a mouse model of metabolic syndrome," Brain, Behavior, and Immunity, vol. 64, pp. 33-49, 2017.

[16] K. Weitkunat, C. Stuhlmann, A. Postel et al., "Short-chain fatty acids and inulin, but not guar gum, prevent diet-induced obesity and insulin resistance through differential mechanisms in mice," Scientific Reports, vol. 7, no. 1, p. 6109, 2017.

[17] C. Chan, C. M. Hyslop, V. Shrivastava, A. Ochoa, R. A. Reimer, and C. Huang, "Oligofructose as an adjunct in treatment of diabetes in NOD mice," Scientific Reports, vol. 6, no. 1, article 37627, 2016.

[18] S. O'Connor, S. Chouinard-Castonguay, C. Gagnon, and I. Rudkowska, "Prebiotics in the management of components of the metabolic syndrome," Maturitas, vol. 104, pp. 11-18, 2017.

[19] A. Aliasgharzadeh, M. Khalili, E. Mirtaheri et al., "A combination of prebiotic inulin and oligofructose improve some of cardiovascular disease risk factors in women with type 2 diabetes: 
a randomized controlled clinical trial," Advanced Pharmaceutical Bulletin, vol. 5, no. 4, pp. 507-514, 2015.

[20] S. Genta, W. Cabrera, N. Habib et al., "Yacon syrup: beneficial effects on obesity and insulin resistance in humans," Clinical Nutrition, vol. 28, no. 2, pp. 182-187, 2009.

[21] N. Roshanravan, R. Mahdavi, E. Alizadeh et al., "Effect of butyrate and inulin supplementation on glycemic status, lipid profile and glucagon-like peptide 1 level in patients with type 2 diabetes: a randomized double-blind, placebo-controlled trial," Hormone and Metabolic Research, vol. 49, no. 11, pp. 886-891, 2017.

[22] J. Luo, M. van Yperselle, S. W. Rizkalla, F. Rossi, F. R. J. Bornet, and $\mathrm{X}$. Slama, "Chronic consumption of short-chain fructooligosaccharides does not affect basal hepatic glucose production or insulin resistance in type 2 diabetics," The Journal of Nutrition, vol. 130, no. 6, pp. 1572-1577, 2000.

[23] N. D. Guess, A. Dornhorst, N. Oliver, J. D. Bell, E. L. Thomas, and G. S. Frost, "A randomized controlled trial: the effect of inulin on weight management and ectopic fat in subjects with prediabetes," Nutrition \& Metabolism, vol. 12, no. 1, p. 36, 2015.

[24] A. Ghavami, N. Roshanravan, S. Alipour et al., "Assessing the effect of high performance inulin supplementation via KLF5 mRNA expression in adults with type 2 diabetes: a randomized placebo controlled clinical trial," Advanced Pharmaceutical Bulletin, vol. 8, no. 1, pp. 39-47, 2018.

[25] B. Pourghassem Gargari, P. Dehghan, A. Aliasgharzadeh, and M. Asghari Jafar-abadi, "Effects of high performance inulin supplementation on glycemic control and antioxidant status in women with type 2 diabetes," Diabetes and Metabolism Journal, vol. 37, no. 2, pp. 140-148, 2013.

[26] P. Dehghan, B. Pourghassem Gargari, and M. Asgharijafarabadi, "Effects of high performance inulin supplementation on glycemic status and lipid profile in women with type 2 diabetes: a randomized, placebo-controlled clinical trial," Health Promotion Perspective, vol. 3, no. 1, pp. 55-63, 2013.

[27] P. Dehghan, M. A. Farhangi, F. Tavakoli, A. Aliasgarzadeh, and A. M. Akbari, "Impact of prebiotic supplementation on $\mathrm{T}$-cell subsets and their related cytokines, anthropometric features and blood pressure in patients with type 2 diabetes mellitus: a randomized placebo-controlled trial," Complementary Therapies in Medicine, vol. 24, pp. 96-102, 2016.

[28] P. Dehghan, B. P. Gargari, M. A. Jafar-Abadi, and A. Aliasgharzadeh, "Inulin controls inflammation and metabolic endotoxemia in women with type 2 diabetes mellitus: a randomized-controlled clinical trial," International Journal of Food Sciences and Nutrition, vol. 65, no. 1, pp. 117-123, 2014.

[29] X. Cai, H. Yu, L. Liu et al., "Milk powder co-supplemented with inulin and resistant dextrin improves glycemic control and insulin resistance in elderly type 2 diabetes mellitus: a 12-week randomized, double-blind, placebo-controlled trial," Molecular Nutrition \& Food Research, vol. 62, no. 24, article 1800865, 2018.

[30] P. Dehghan, B. Pourghassem Gargari, and M. Asghari Jafar-abadi, "Oligofructose-enriched inulin improves some inflammatory markers and metabolic endotoxemia in women with type 2 diabetes mellitus: a randomized controlled clinical trial," Nutrition, vol. 30, no. 4, pp. 418-423, 2014.

[31] M. A. Farhangi, A. Z. Javid, and P. Dehghan, "The effect of enriched chicory inulin on liver enzymes, calcium homeostasis and hematological parameters in patients with type 2 diabetes mellitus: a randomized placebo-controlled trial," Primary Care Diabetes, vol. 10, no. 4, pp. 265-271, 2016.

[32] Z. Asemi, S. A. Alizadeh, K. Ahmad, M. Goli, and A. Esmaillzadeh, "Effects of beta-carotene fortified synbiotic food on metabolic control of patients with type 2 diabetes mellitus: a double-blind randomized cross-over controlled clinical trial," Clinical Nutrition, vol. 35, no. 4, pp. 819825, 2016.

[33] M. Tajadadi-Ebrahimi, F. Bahmani, H. Shakeri et al., "Effects of daily consumption of synbiotic bread on insulin metabolism and serum high-sensitivity C-reactive protein among diabetic patients: a double-blind, randomized, controlled clinical trial," Annals of Nutrition \& Metabolism, vol. 65, no. 1, pp. 34-41, 2014.

[34] M. Tajabadi-Ebrahimi, N. Sharifi, A. Farrokhian et al., "A randomized controlled clinical trial investigating the effect of synbiotic administration on markers of insulin metabolism and lipid profiles in overweight type 2 diabetic patients with coronary heart disease," Experimental and Clinical Endocrinology \& Diabetes, vol. 125, no. 01, pp. 21-27, 2017.

[35] N. K. A. Bonsu and S. Johnson, "Effects of inulin fiber supplementation on serum glucose and lipid concentration in patients with type 2 diabetes," Diabetes \& Metabolism, vol. 21, pp. 80-86, 2012.

[36] M. S. Alles, N. M. de Roos, J. C. Bakx, E. van de Lisdonk, P. L. Zock, and J. G. A. J. Hautvast, "Consumption of fructooligosaccharides does not favorably affect blood glucose and serum lipid concentrations in patients with type 2 diabetes," The American Journal of Clinical Nutrition, vol. 69, no. 1, pp. 64-69, 1999.

[37] N. D. Guess, A. Dornhorst, N. Oliver, and G. S. Frost, "A randomised crossover trial: the effect of inulin on glucose homeostasis in subtypes of prediabetes," Annals of Nutrition \& Metabolism, vol. 68, no. 1, pp. 26-34, 2016.

[38] C. J. Rebello, J. Burton, M. Heiman, and F. L. Greenway, "Gastrointestinal microbiome modulator improves glucose tolerance in overweight and obese subjects: a randomized controlled pilot trial," Journal of Diabetes and its Complications, vol. 29, no. 8, pp. 1272-1276, 2015.

[39] E. M. Dewulf, P. D. Cani, S. P. Claus et al., "Insight into the prebiotic concept: lessons from an exploratory, double blind intervention study with inulin-type fructans in obese women," Gut, vol. 62, no. 8, pp. 1112-1121, 2013.

[40] N. M. Daud, N. A. Ismail, E. L. Thomas et al., "The impact of oligofructose on stimulation of gut hormones, appetite regulation and adiposity," Obesity, vol. 22, no. 6, pp. 1430-1438, 2014.

[41] D. A. de Luis, B. de la Fuente, O. Izaola, R. Aller, S. Gutiérrez, and M. Morillo, "Double blind randomized clinical trial controlled by placebo with a fos enriched cookie on saciety and cardiovascular risk factors in obese patients," Nutrición Hospitalaria, vol. 28, no. 1, pp. 78-85, 2013.

[42] J. A. Parnell and R. A. Reimer, "Weight loss during oligofructose supplementation is associated with decreased ghrelin and increased peptide YY in overweight and obese adults," The American Journal of Clinical Nutrition, vol. 89, no. 6, pp. 1751-1759, 2009.

[43] A. R. Tovar, M. C. Caamaño, S. Garcia-Padilla, O. P. García, M. A. Duarte, and J. L. Rosado, "The inclusion of a partial meal replacement with or without inulin to a calorie restricted diet contributes to reach recommended intakes of micronutrients and decrease plasma triglycerides: a randomized clinical trial 
in obese Mexican women," Nutrition Journal, vol. 11, no. 1, p. $44,2012$.

[44] M. Laakso and J. Kuusisto, "Insulin resistance and hyperglycaemia in cardiovascular disease development," Nature Reviews Endocrinology, vol. 10, no. 5, pp. 293-302, 2014.

[45] G. J. Biessels and L. P. Reagan, "Hippocampal insulin resistance and cognitive dysfunction," Nature Reviews Neuroscience, vol. 16, no. 11, pp. 660-671, 2015.

[46] D. Athauda and T. Foltynie, "Insulin resistance and Parkinson's disease: a new target for disease modification?," Progress in Neurobiology, vol. 145-146, pp. 98-120, 2016.

[47] F. Brighenti, "Dietary fructans and serum triacylglycerols: a meta-analysis of randomized controlled trials," The Journal of Nutrition, vol. 137, no. 11, pp. 2552S-2556S, 2007.

[48] L. Collado Yurrita, I. San Mauro Martín, M. J. CiudadCabañas, M. E. Calle-Purón, and M. Hernández Cabria, "Effectiveness of inulin intake on indicators of chronic constipation; a meta-analysis of controlled randomized clinical trials," Nutrición Hospitalaria, vol. 30, no. 2, pp. 244-252, 2014.

[49] A. C. Apolinário, B. P. G. de Lima Damasceno, N. E. de Macêdo Beltrão, A. Pessoa, A. Converti, and J. A. da Silva, "Inulin-type fructans: a review on different aspects of biochemical and pharmaceutical technology," Carbohydrate Polymers, vol. 101, pp. 368-378, 2014.

[50] G. Flamm, W. Glinsmann, D. Kritchevsky, L. Prosky, and M. Roberfroid, "Inulin and oligofructose as dietary fiber: a review of the evidence," Critical Reviews in Food Science and Nutrition, vol. 41, no. 5, pp. 353-362, 2001.

[51] P. D. Cani and N. M. Delzenne, "The role of the gut microbiota in energy metabolism and metabolic disease," Current Pharmaceutical Design, vol. 15, no. 13, pp. 1546-1558, 2009.

[52] C. Cherbut, "Motor effects of short-chain fatty acids and lactate in the gastrointestinal tract," The Proceedings of the Nutrition Society, vol. 62, no. 1, pp. 95-99, 2003.

[53] S. Khan and G. B. Jena, "Protective role of sodium butyrate, a HDAC inhibitor on beta-cell proliferation, function and glucose homeostasis through modulation of p38/ERK MAPK and apoptotic pathways: study in juvenile diabetic rat," Chemico-Biological Interactions, vol. 213, pp. 1-12, 2014.

[54] J. Tian, H. N. Dang, J. Yong et al., "Oral treatment with $\gamma$-aminobutyric acid improves glucose tolerance and insulin sensitivity by inhibiting inflammation in high fat diet-fed mice," PLoS One, vol. 6, no. 9, article e25338, 2011.

[55] F. De Vadder, P. Kovatcheva-Datchary, D. Goncalves et al., "Microbiota-generated metabolites promote metabolic benefits via gut-brain neural circuits," Cell, vol. 156, no. 1-2, pp. 84-96, 2014.

[56] E. Esteve, W. Ricart, and J. M. Fernandez-Real, "Gut microbiota interactions with obesity, insulin resistance and type 2 diabetes: did gut microbiote co-evolve with insulin resistance?," Current Opinion in Clinical Nutrition and Metabolic Care, vol. 14, no. 5, pp. 483-490, 2011.

[57] H. Yadav, J. H. Lee, J. Lloyd, P. Walter, and S. G. Rane, "Beneficial metabolic effects of a probiotic via butyrate-induced GLP-1 hormone secretion," The Journal of Biological Chemistry, vol. 288, no. 35, pp. 25088-25097, 2013.

[58] M. Diamant, E. E. Blaak, and W. M. de Vos, "Do nutrient-gutmicrobiota interactions play a role in human obesity, insulin resistance and type 2 diabetes?," Obesity Reviews, vol. 12, no. 4, pp. 272-281, 2011.
[59] A. M. Caricilli, P. K. Picardi, L. L. de Abreu et al., "Gut microbiota is a key modulator of insulin resistance in TLR 2 knockout mice," PLoS Biology, vol. 9, no. 12, article e1001212, 2011.

[60] N. Roshanravan, R. Mahdavi, E. Alizadeh et al., "The effects of sodium butyrate and inulin supplementation on angiotensin signaling pathway via promotion of Akkermansia muciniphila abundance in type 2 diabetes; a randomized, double-blind, placebo-controlled trial," Journal of Cardiovascular and Thoracic Research, vol. 9, no. 4, pp. 183-190, 2017.

[61] American Diabetes Association, "Classification and diagnosis of diabetes," Diabetes Care, vol. 40, Supplement 1, pp. S11S24, 2017. 


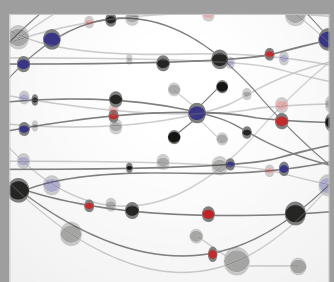

The Scientific World Journal
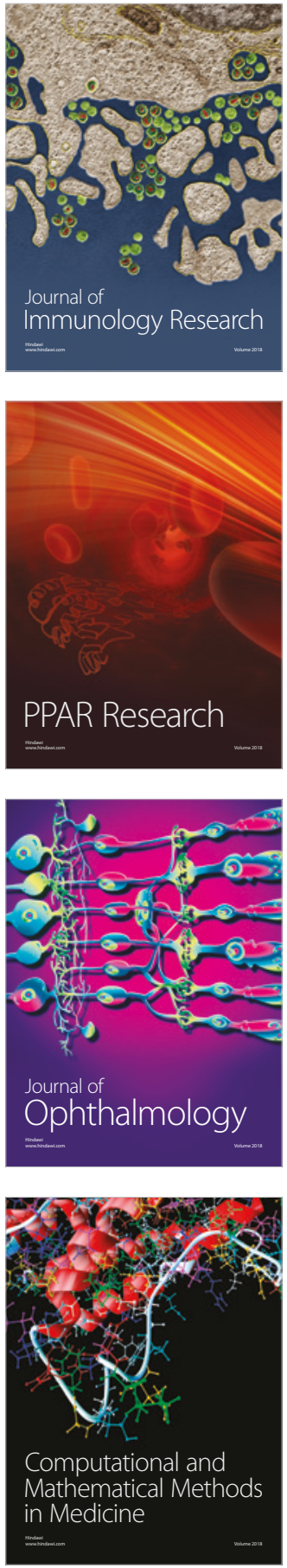

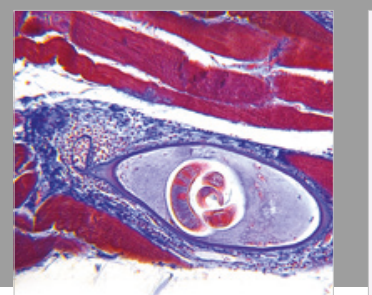

Gastroenterology Research and Practice

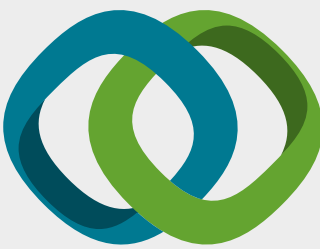

\section{Hindawi}

Submit your manuscripts at

www.hindawi.com
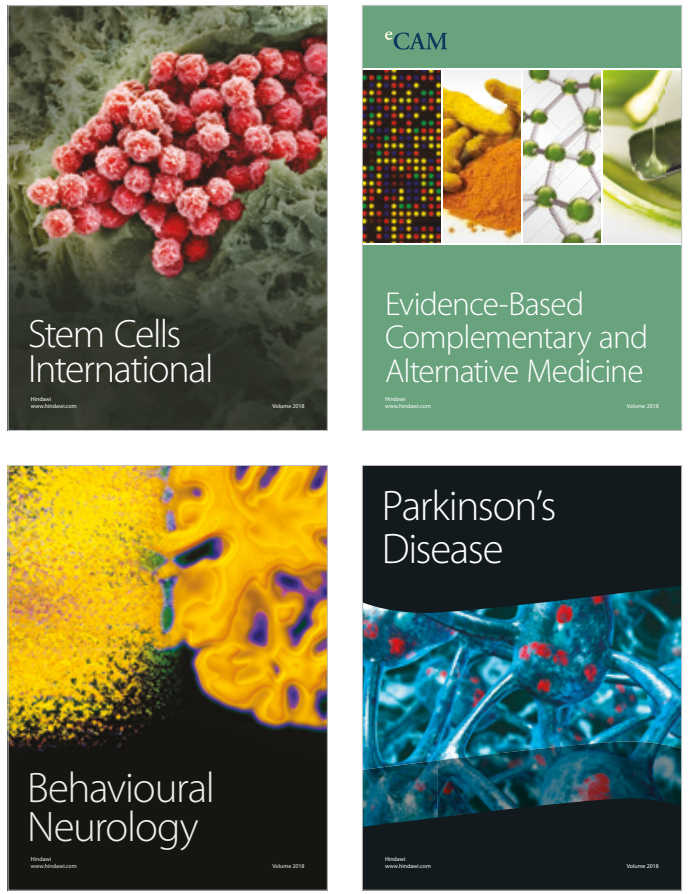

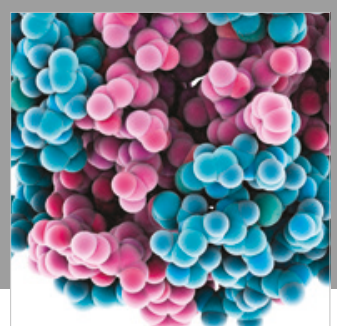

ournal of

Diabetes Research

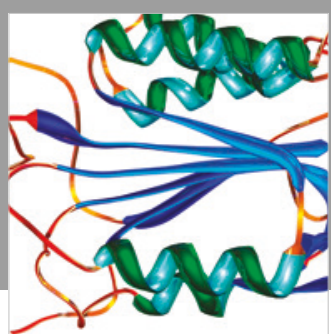

Disease Markers
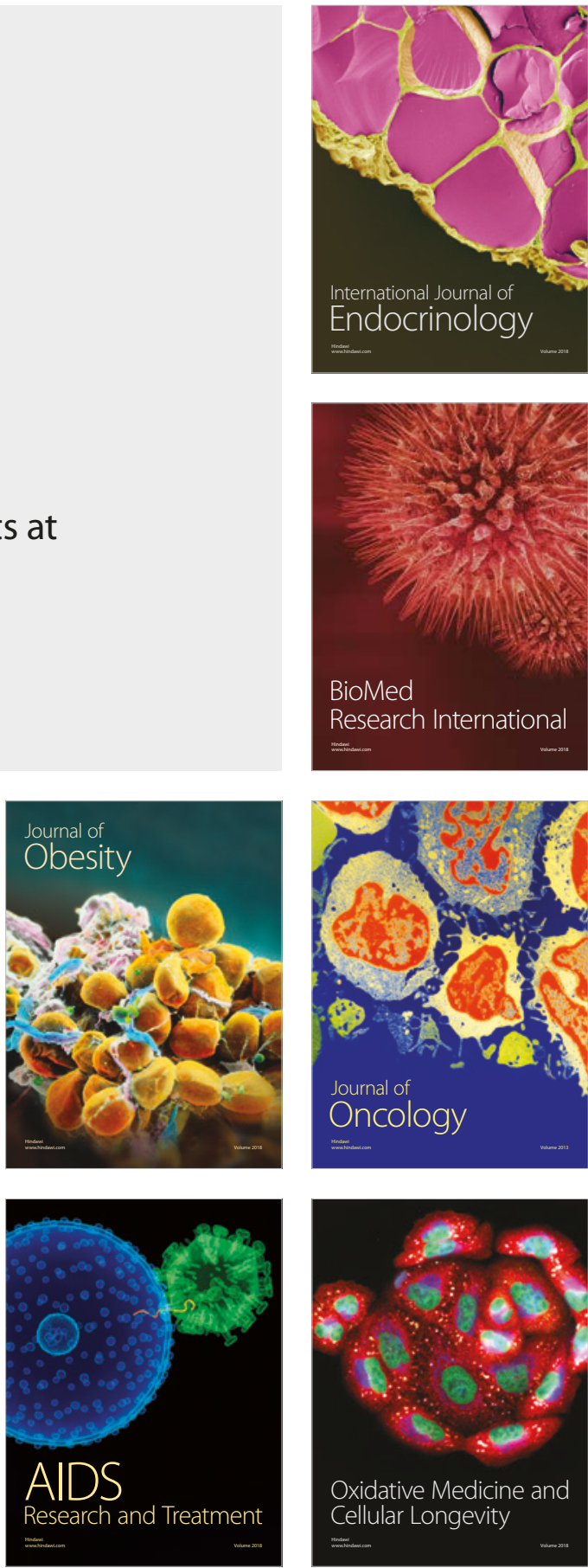